\title{
Design and Implementation of the Boundary Layer Transition Flight Experiment on Space Shuttle Discovery
}

\author{
Theodoros A. Spanos ${ }^{1}$, and Ann Micklos ${ }^{2}$ \\ United Space Alliance, Cape Canaveral, FL 32920
}

\begin{abstract}
In an effort to better the understanding of high speed aerodynamics, a series of flight experiments were installed on Space Shuttle Discovery during the STS-119 and STS-128 missions. This experiment, known as the Boundary Layer Transition Flight Experiment (BLT FE), provided the technical community with actual entry flight data from a known height protuberance at Mach numbers at and above Mach 15. Any such data above Mach 15 is irreproducible in a laboratory setting. Years of effort have been invested in obtaining this valuable data, and many obstacles had to be overcome in order to ensure the success of implementing an Orbiter modification. Many Space Shuttle systems were involved in the installation of appropriate components that revealed 'concurrent engineering' was a key integration tool. This allowed the coordination of all various parts and pieces which had to be sequenced appropriately and installed at the right time. Several issues encountered include Orbiter configuration and access, design requirements versus current layout, implementing the modification versus typical processing timelines, and optimizing the engineering design cycles and changes. Open lines of communication within the entire modification team were essential to project success as the team was spread out across the United States, from NASA Kennedy Space Center in Florida, to NASA Johnson Space Center in Texas, to Boeing Huntington Beach, California among others. The forum permits the discussion of processing concerns from the design phase to the implementation phase, which eventually saw the successful flights and data acquisition on STS-119 in March 2009 and on STS-128 in September 2009.
\end{abstract}

\section{Introduction}

The primary reason regarding why the Boundary Layer Transition Flight Experiment (BLT FE) is taking place at the end of the Space Shuttle Program (SSP) is to better the understanding of high Mach number / high enthalpy non-equilibrium condition entry aerothermodynamics. One key motivator for the SSP to pursue the BLT FE data was the event noticed by the rendezvous pitch maneuver (RPM) during STS-114. Photographs taken of Space Shuttle Discovery's lower surface revealed two locations of protruding gap fillers. Analysis conducted at the Johnson Space Center in Houston, Texas using thermal modeling methods appropriate at the time, revealed an unsafe condition for reentry existed on one gap filler. Therefore, the SSP decided to send Astronaut Steve Robinson on an Extra Vehicular Activity (EVA) to remove the Ames gap filler. If there had been a better understanding of how a protrusion affects boundary layer transition, then a more accurate analysis could have been performed which could have prevented the dangerous EVA.

"Boundary layer transition during hypersonic entry is one of several fundamental aerothermodynamic effects that influence the design and operation of manned spacecraft. Flight data acquired during the history of the Space Shuttle Program has provided important information regarding the boundary layer transition onset and turbulent heating levels. However, the existing reentry boundary layer transition data on the Orbiter have been obtained as a result of uncontrolled changes in vehicle configuration mainly due to protruding gap fillers. Efforts to develop an approach for performing entry boundary layer transition flight testing have led to the formation of project activity intent on acquiring hypersonic Orbiter flight data." ${ }^{3}$

\footnotetext{
${ }^{1}$ Thermal Protection Systems Engineer, USK-443

${ }^{2}$ Project Manager/Thermal Protection Systems Engineer, USK-306
} 


\section{Design Consideration / Project Overview}

In order to perform this flight test, a protuberance of fixed geometry was required to be installed under the port wing of Discovery with thermocouples installed downstream. The protuberance tile location was chosen with respect to several requirements which allowed for the correct format of data to be gathered. ${ }^{3}$ First and foremost was the concern for the safety of the Crew and Orbiter, therefore, the protuberance was placed in a location where local and downstream heating would not damage the Thermal Protection System (TPS) and any underlying structure. In terms of design, there should be as long and smooth undisturbed flow from the stagnation point to the trip location as possible, as well as a significant run downstream of the protuberance for turbulent flow to start.

Early on in the project, the BLT team proposed to implement an actuated protuberance that could be deployed and retracted. This design would allow the gathering of flight data at various entry Mach numbers. The protuberance's location would be placed along the Orbiter's centerline, just aft and between the main landing gear doors. ${ }^{3}$ The proposed location would give analysts a long flow run from the nose cap to the aft of the vehicle. Unfortunately, the Orbiter Structures Design group was adamant that the BLT project would not put holes into the aft fuselage structure area for instrumentation wire runs. The decision resulted in relocating the protuberance to the left wing. Although the flow field is not as smooth on the wing as it is on the centerline, there still is a long flow run to a stagnation point if the protuberance stays inside the bow shock. The BLT team chose a location that had both the surface area and was robust enough to hold the actuating system on the honeycomb wing skin section outboard of the main landing gear door. However, relocating the protuberance to the wing presented several additional problems: the elevon hinge line is not as thermally robust as the body flap region; therefore additional analysis was requested in the hinge line area to understand the potential over-temperature conditions and minimize concerns. ${ }^{3}$ Another issue was determining a wire path for the actuator system to the starboard side of the Orbiter where the recording system and actuator system originated. Abandoned wiring was identified which ran underneath the payload bay solved this problem for the wire path, but the path for the actuator to the control mechanism in the crew cabin could not be determined. Additionally, the cost of the deployable system was found to be prohibitive as well as the time to manufacture and implement this design. Therefore, a fixed protrusion was selected for the flight experiment. The final protuberance location chosen was on the Orbiter's left wing skin stringer area that maximized the thermal mass under the protuberance tile, while staying within the bow shock region. ${ }^{3}$

The flight experiment has three targeted transition Mach numbers: 15, 18, and 19. Scientists and engineers are capable of producing data using wind tunnels to around Mach 15 which agree with theoretical modeling. At those conditions data is highly theoretical and is primarily Computational Fluid Dynamics (CFD) based. ${ }^{3}$ The first flight targeted a transition in which there was high confidence in the projected results since the protuberance first flight height ( 0.25 inches) was within the Orbiter's flight experience. The second flight targeted an upper limit of transition experienced by the Space Shuttle Orbiter (Mach 18 on STS-28). The data received during STS-28 was very limited and was determined based on post-flight observation of the surrounding area of a protruding gap filler in which the gap filler's exposed height and geometry were not known prior to entry. ${ }^{3}$ The third flight would target a transition outside of the experience base and provide a set of data new to the Space Shuttle Program.

During the first flight test on STS-119, the protuberance height of 0.25 inches was exposed to free-stream airflow. Analysis showed that this height would cause the transition of the boundary layer from laminar to turbulent at around Mach 15. When the results of the first flight experiment were obtained and analyzed, the team felt confident that installing a taller (more exposed to the free-stream airflow) 0.35 inch protuberance would yield transition at higher Mach numbers (Mach 18). ${ }^{3}$ Results of the second (STS-128) experiment are being analyzed at the time of the authoring of this paper with the ultimate goal of installing a protuberance that would achieve a transition at Mach 19. Understanding this realm of hypersonic aerodynamics will allow for a less conservative (and more accurate) modeling method for future vehicles for NASA, the Air Force, and private industry. ${ }^{3}$ 


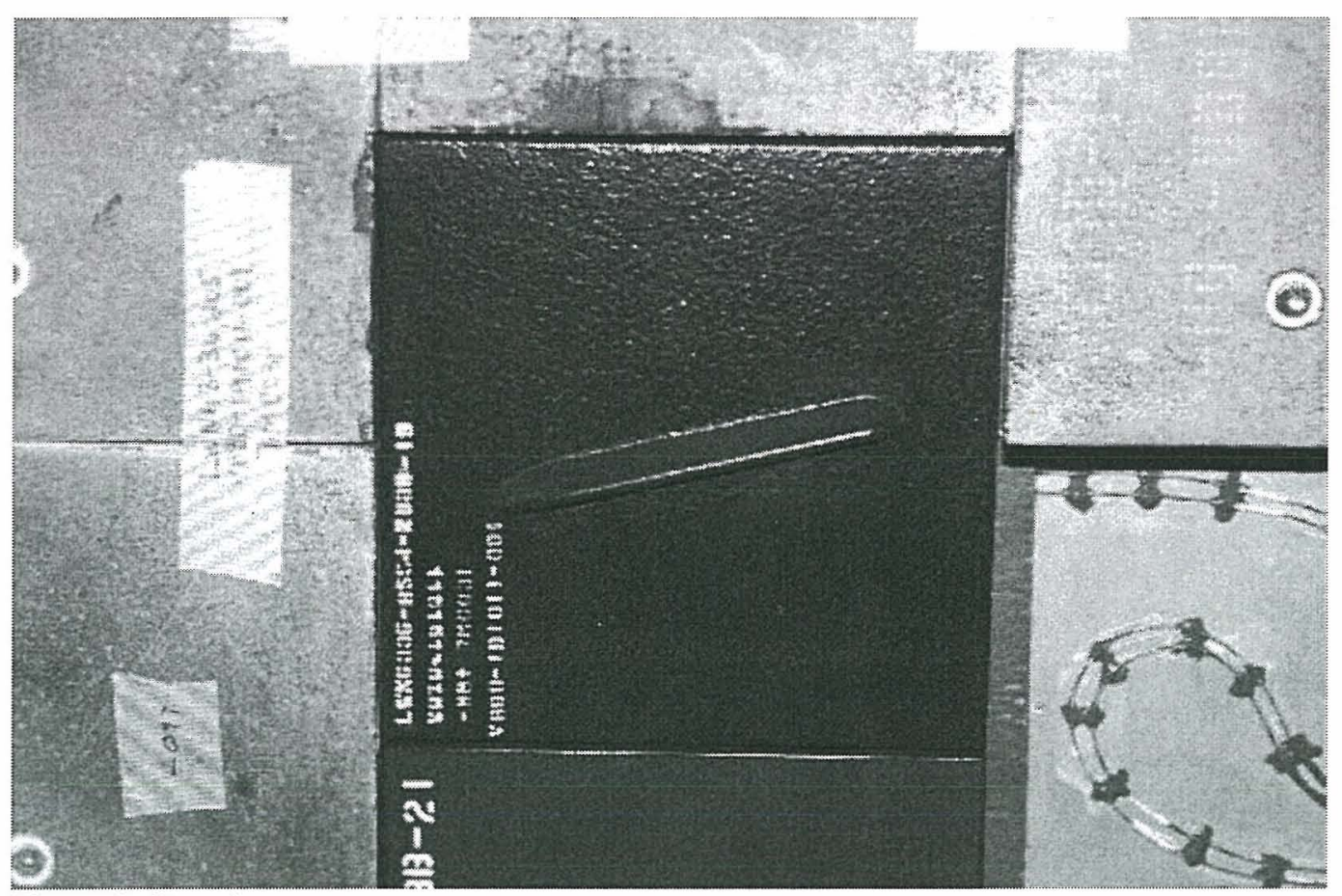

Figure 1. Protuberance

\section{Airframe Structure / Thermal Protection System}

Two symbiotic systems on the Space Shuttle Orbiter are the Airframe Structure and the Thermal Protection System. The airframe will not be able to survive the searing heat of atmospheric re-entry without the TPS, and TPS bonds its components to the airframe structure. This relationship played a key role in the implementation of the BLT FE. A brief overview of both systems is appropriate as the layout of the structural components determined subsequent installation procedures.

The airframe structure in the port wing, which is the region of concern for BLT FE purposes, is very similar in design to modern aircraft; it is composed primarily of an aluminum alloy (and a series of spars run along the longitudinal axis, on to which a series of ribs that run along the lateral axis connect to. The aluminum honeycomb structure is further bolstered by a series of stringers which also run along the lateral axis). ${ }^{7}$

The structures system had to be modified accommodate the BLT experiment. First, the structural penetrations had to be created in the wing-skin to allow for thermocouple wire installation. ${ }^{7}$ Second, NonDestructive Engineering (NDE) had to be performed, without having an adverse effect to the TPS or structural system, and accurately locate the Orbiter coordinates of the penetration holes. Third, temperature indication labels had to be installed internal to the wing in specific areas near the penetration holes. Fourth, an area had to be chosen that could support the installation of the Thermocouple Reference Junction (TRJ), a device that allows the input of ten thermocouples. ${ }^{6}$

The Thermal Protection System (TPS) is the only system on the Space Shuttle Orbiter that does not have a redundant backup. It is also responsible for dissipating all of the re-entry heating, thus protecting the underlying airframe structure. The TPS is made up of many components, each of which has a unique installation procedure. However, for the purposes of this discussion, the focus will be on the lower surface of the Orbiter which is composed of approximately 22,000 High Temperature Reusable Surface Insulation (HRSI) tiles. The TPS was designed with the following criteria in mind:

"The inner airframe structure must be maintained at a temperature less than $350 \mathrm{~F}$ with a maximum outer surface temperature of $2300 \mathrm{~F}$, and must be reusable under these conditions for 100 missions. They must also match the contour of the airframe structure and maintain an aerodynamically smooth outer surface. Low density materials must be used that are able to withstand stresses and vibrations that are produced during launch, and thermally induced stresses during temperature changes $(-170 \mathrm{~F}$ to $2300 \mathrm{~F})$. Finally, they must experience a minimum amount of absorption of moisture and other contaminants and must also adhere to the airframe structure." 4

HRSI tiles come in several different varieties based on density. The three types of tiles used in the BLT FE were LI-900, LI2200 , and BRI-18 each corresponding to densities of 9,22 , and $18 \mathrm{lbm} / \mathrm{cu} \mathrm{ft}$ respectively. ${ }^{7}$ 
The sub-installations of filler-bar, and the Strain Isolation Pad (SIP) are also required to support a tile installation. Filler bar is a thin strip of Nomex felt material that has dimensional stability at high temperatures. It is bonded with Room Temperature Vulcanizing Silicone Adhesive (RTV) to the structure to create a thermal barrier in the gaps between tiles. ${ }^{7}$ SIP is a non rigid piece of Nomex polyaramid felt pad used to compensate for the differences in thermal expansion coefficient between the tiles and the aluminum substrate and to provide vibration isolation. SIP comes in three varieties, each corresponding to a different thickness. ${ }^{7}$ The 0.090 inch SIP is used for high modulus bonding applications (typically next to stiffer components such as thermal barrier installations). The other two are 0.115 inch and 0.160 inch SIP, the latter of which is the most common and is used in acreage tile bonds. A typical bonding installation is shown in Figure 2.

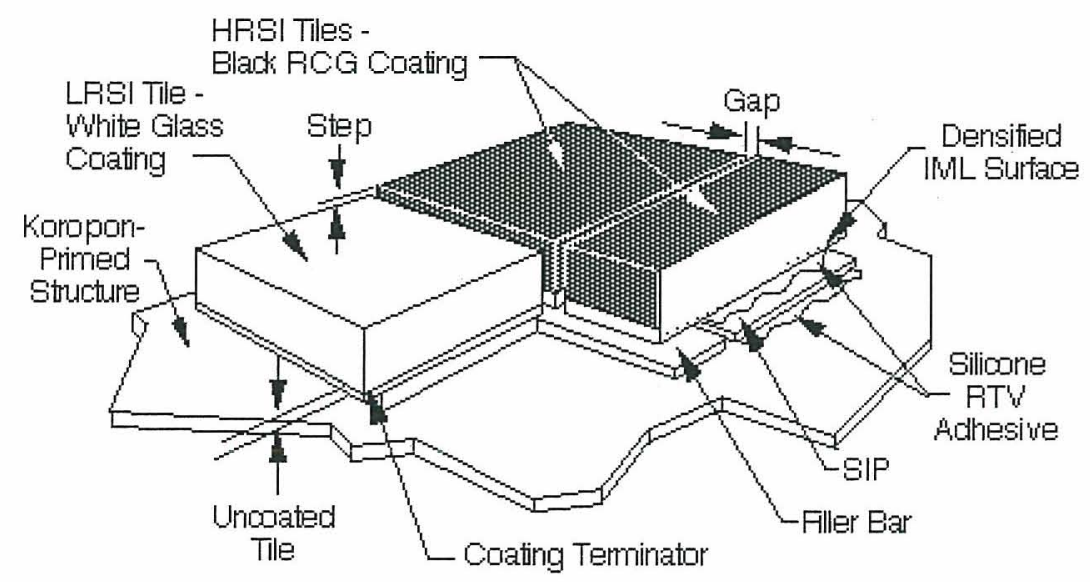

Figure 2. Typical Tile Bond Installation

In order to implement the experiment on the Orbiter, a series of tiles had to be removed. The substrate then had to be cleaned and primed for the new tile installations. Two types of tiles were used: Instrumented and

Non-Instrumented (tiles that have thermocouples installed, and tiles that do not). ${ }^{5}$ At this juncture, the discussion will focus on non-instrumented tiles. A typical tile is processed through a series of prefits which check that the tile meets the limits of how far it is displaced vertically and horizontally with respect to the adjacent installed tile. This is considered the 'step and gap' criteria in the TPS community. ${ }^{7}$ A second test for a standard tile installation deals with checking the mismatch between the tile Inner Mold Line (IML) and the airframe structure. Mismatch checks to see that there will not be a void between the two, thereby compromising the bond area. When the step and gap criteria and mismatch are met (among other checks) the tile is bonded with RTV to the Orbiter. ${ }^{7}$

The inclusion of a thermocouple in the tile modifies the installation sequences. The tile is received by engineering with thermocouple wire leads protruding from the IML. Recess pockets are machined in the IML which will eventually provide a small pocket for the RTV to pool and act as a locking mechanism when the leads are spliced to extension wires (Figure 3). A series of functional tests (test of resistance and polarity) are performed before and after the extension wires are routed internally (Figure 4) and attached to the Thermocouple Reference Junction (TRJ). ${ }^{7}$ These tests verify that the shipside wires match the polarity of the thermocouple wires. 


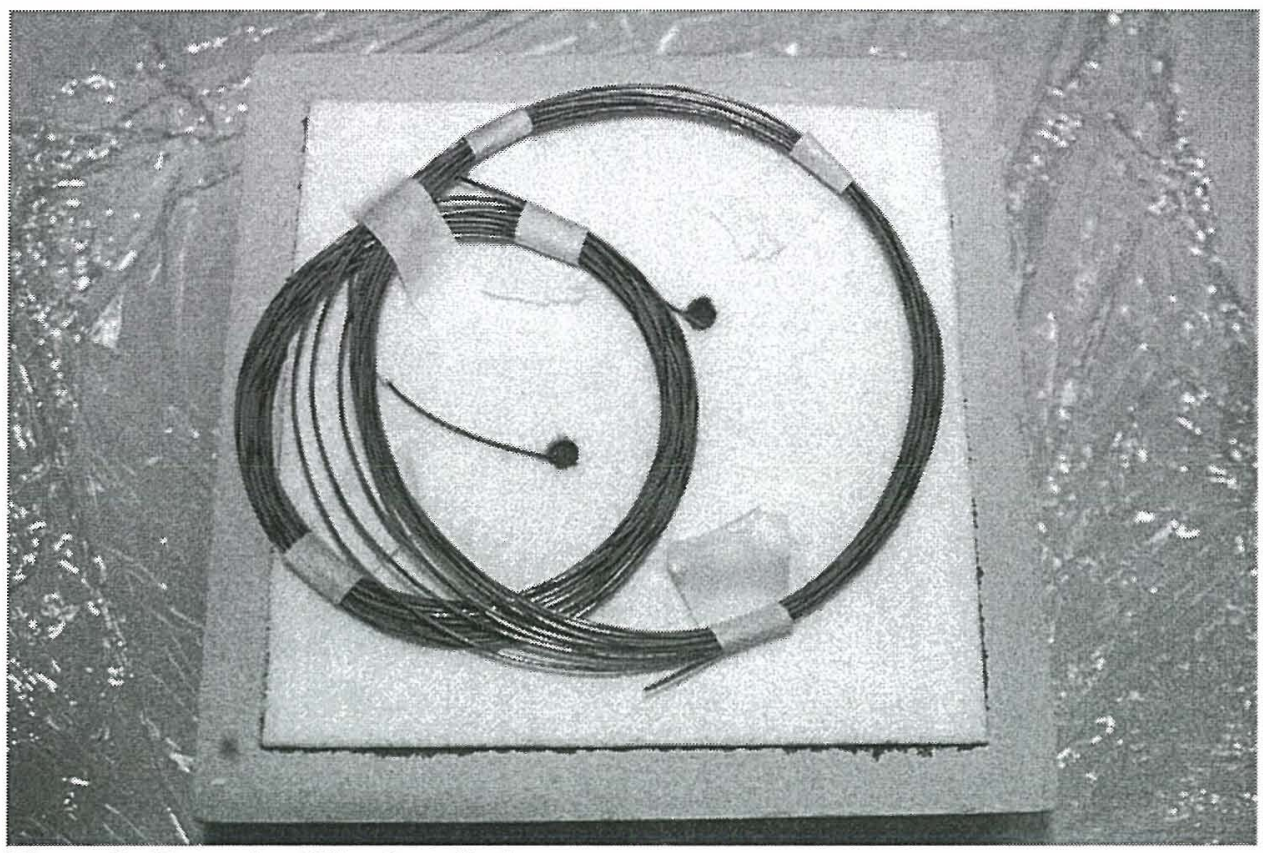

Figure 3. Extension Wire Installation

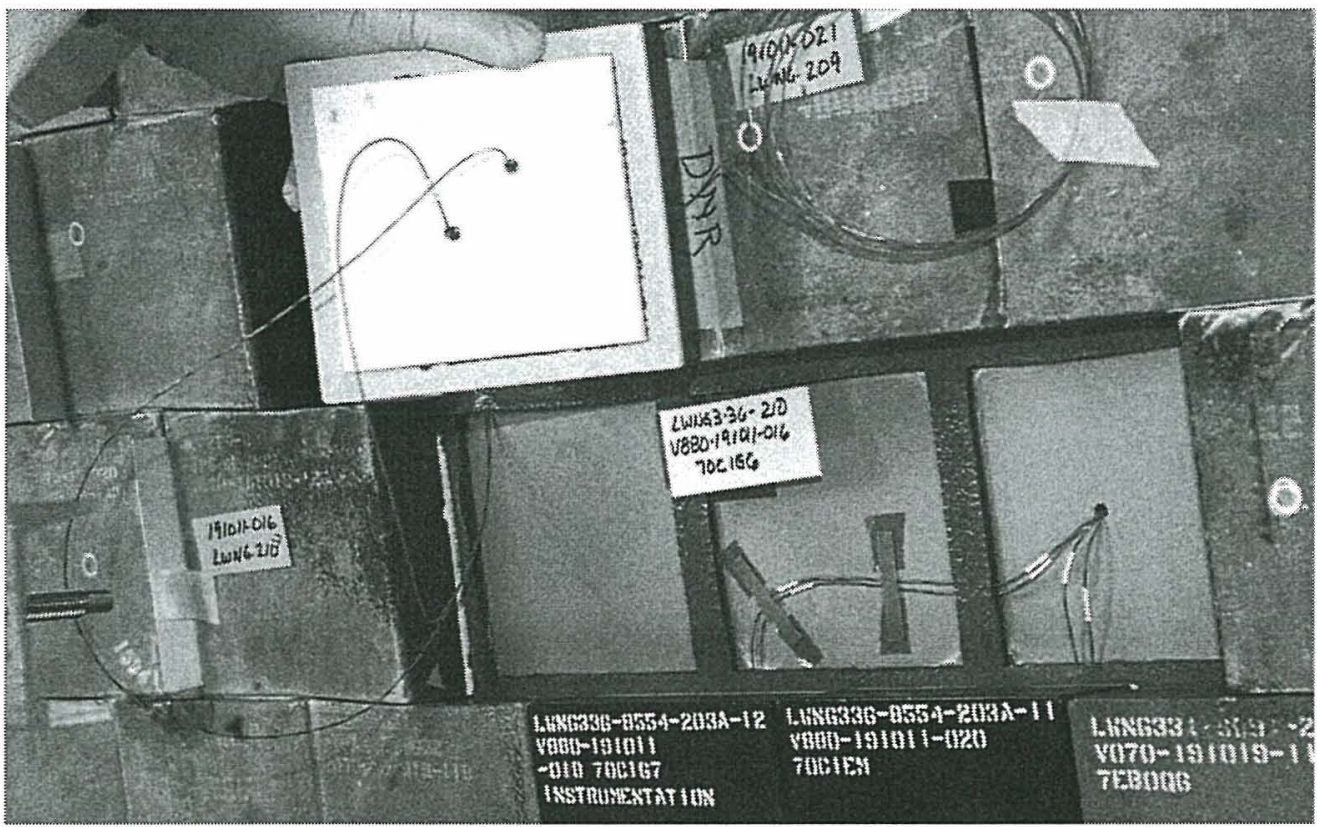

Figure 4. Routing Thermocouple Wires Internally

The BLT FE is unique in that a multitude of tiles had to be processed and sequenced appropriately. Twenty one tiles were removed from the lower port wing in direct support of BLT FE first flight (three additional tiles were removed in order to support installation of the flight experiment). ${ }^{5}$ The twenty one tiles are highlighted in yellow in Figure 5 and are listed in Table 1 for the first flight and Table 2 for the second flight. As the airflow moved downstream from the control, it encounters the protuberance and the experiment begins in earnest. For the first flight, the protuberance measured 0.25 inches tall and 4.0 inches long. ${ }^{6}$ There was a thermocouple installed in the fin of the protuberance itself (Figure 1) which recorded what was expected to be the peak temperature of the experiment. The two tiles adjacent to the protuberance (V880-191011-040 \& V880-191011-041) as well as the V880-191011-036 were removed and replaced in order to bolster the thermal capabilities in the area. By installing a 
LI-2200 tile in place of an LI-900, an increase in silica density corresponded to an ability of the area to withstand the higher heat fluxes produced by the installation of the protuberance. ${ }^{5}$

Extensive coordination of multiple systems was required in order to achieve the appropriate bond sequencing. The structures engineers had to have the boreholes drilled in the aluminum skin of the Orbiter first, then install the grommet components. ${ }^{5}$ Concurrently, the TRJ had to be installed and checked out. Orbiter electrical engineers had to install the wire runs from the TRJ to the MADS in order to connect the two. ${ }^{7}$ Meanwhile, the instrumentation engineers' responsibility was to checkout those components and when the thermal engineers were ready to install the tiles, checkout and channelize the thermocouple installation from an individual thermocouple and its response to the MADS (end-to-end test). ${ }^{7}$ When all components were installed and checked out, a preflight 3-D scan was taken of the protuberance and surrounding tiles. This gave analysts and engineers a baseline geometry. Had the geometry changed during flight, analysts would need to account for the difference. As such a post-flight image was also required.

STS-119 launched on 15 March 2009 and had early success. Engineers were able to review data from the MADS while the mission was ongoing that revealed the MADS recorded ascent heating as expected. Entry data revealed that all ten thermocouples performed as expected. The first indications of boundary layer transition from laminar to turbulent flow occurred at TC\#1 (Figure 5) which is the aft and outboard most thermocouple. Transition was seen at Mach $15.6 \pm 0.1$ at 969 seconds with the protuberance becoming turbulent at Mach $7.71 \pm 0.3$ at 1224 seconds. Both were very close to the predicted values. However, the temperature experienced by the protuberance was approximately 2000 degrees Fahrenheit, much less than the predicted temperature of 2900 degrees Fahrenheit. A 3-D comparison of preflight and post-flight images of the protuberance and surrounding geometry revealed no significant change had occurred, nor was there an adverse affect on the downstream tiles within the turbulent wedge region. Figures 5 through 8 and Table 10-1 show the location of the protuberance, properties of the tiles, and active thermocouples for STS-119. 
Boundary Laver Transition Flight Experiment STS-119 OV-103 FLT-36 Layout
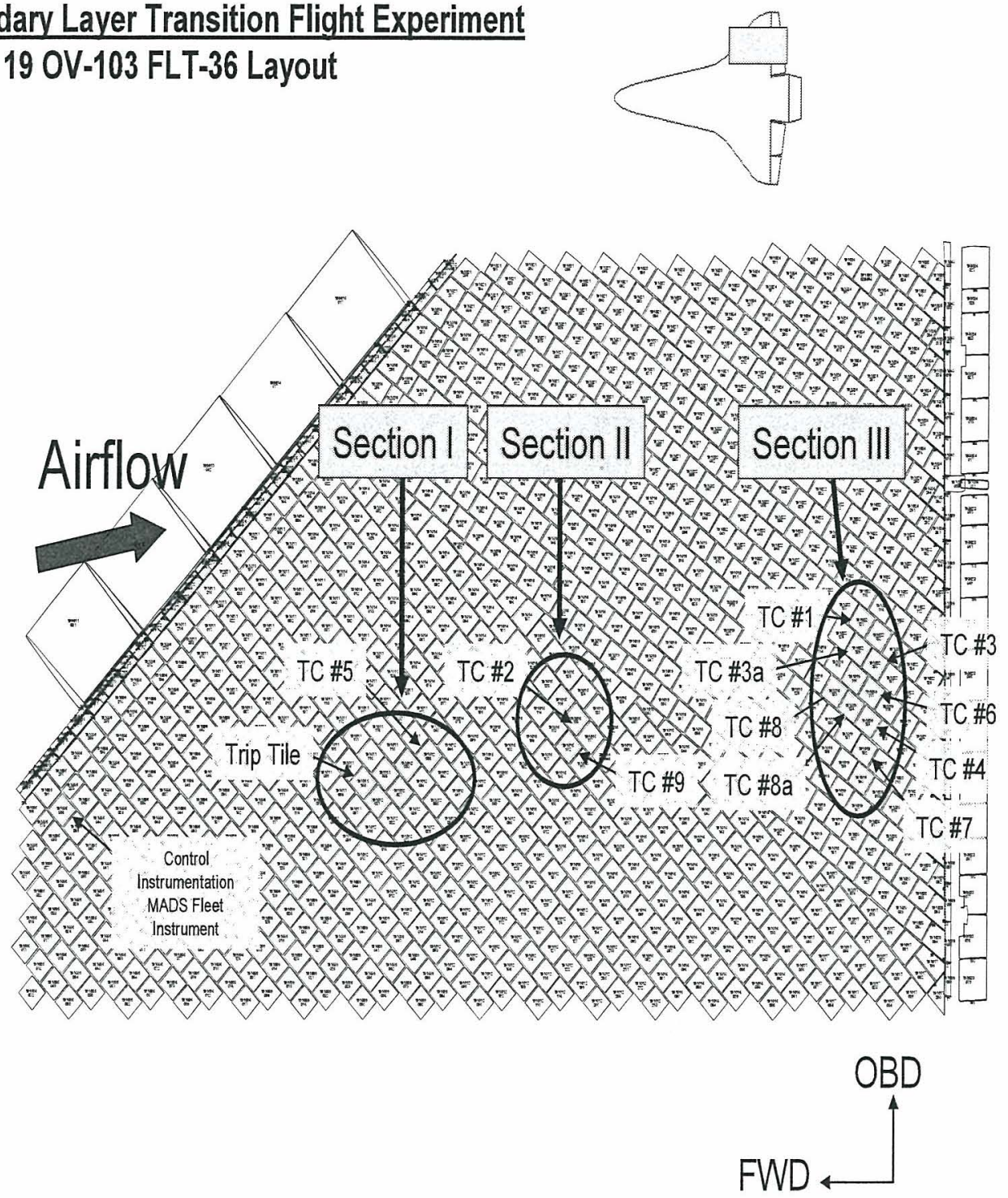

Figure 5. Tile Layout for STS-119 


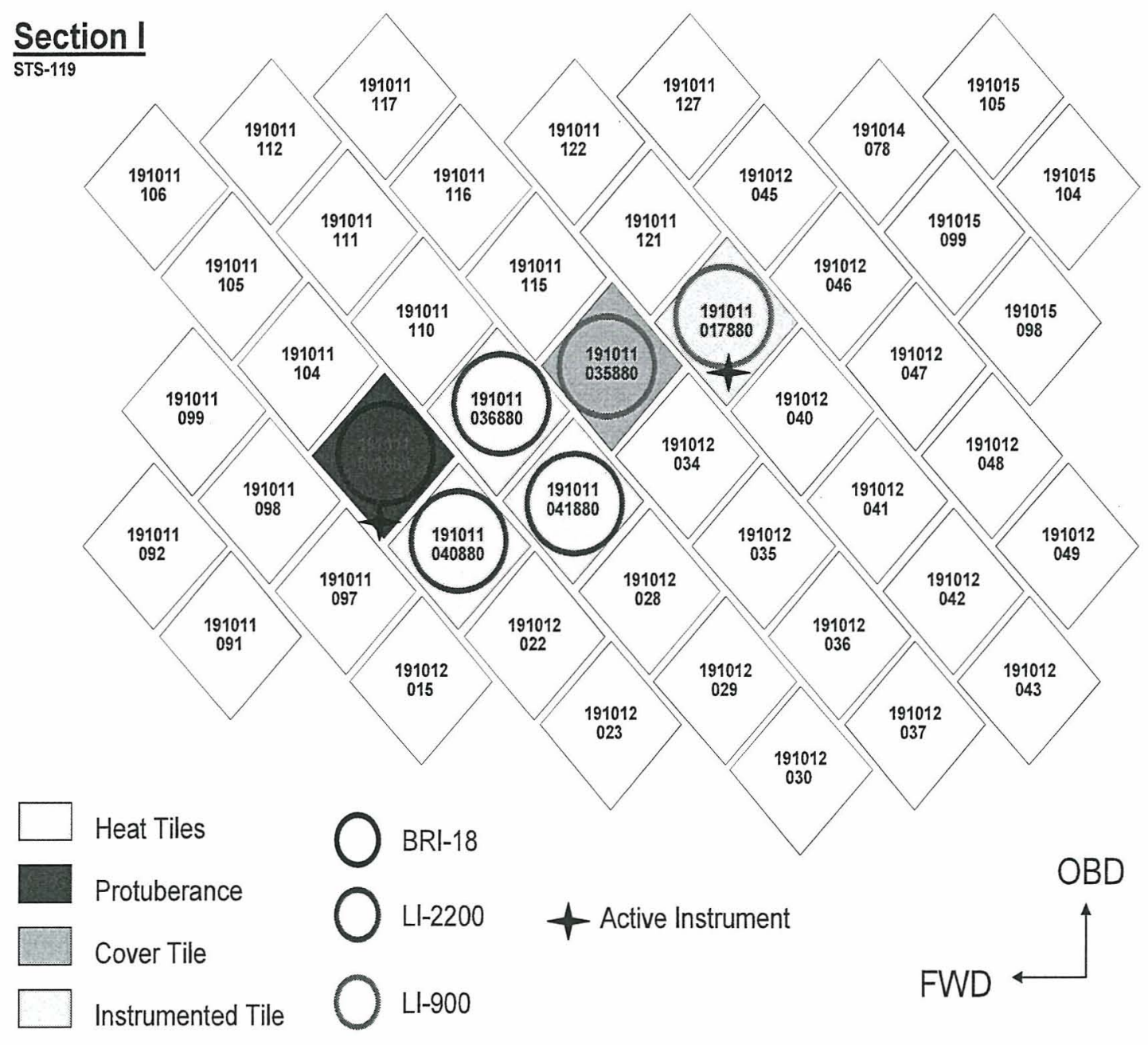

Figure 6. Detailed View of STS-119 Section I Layout 


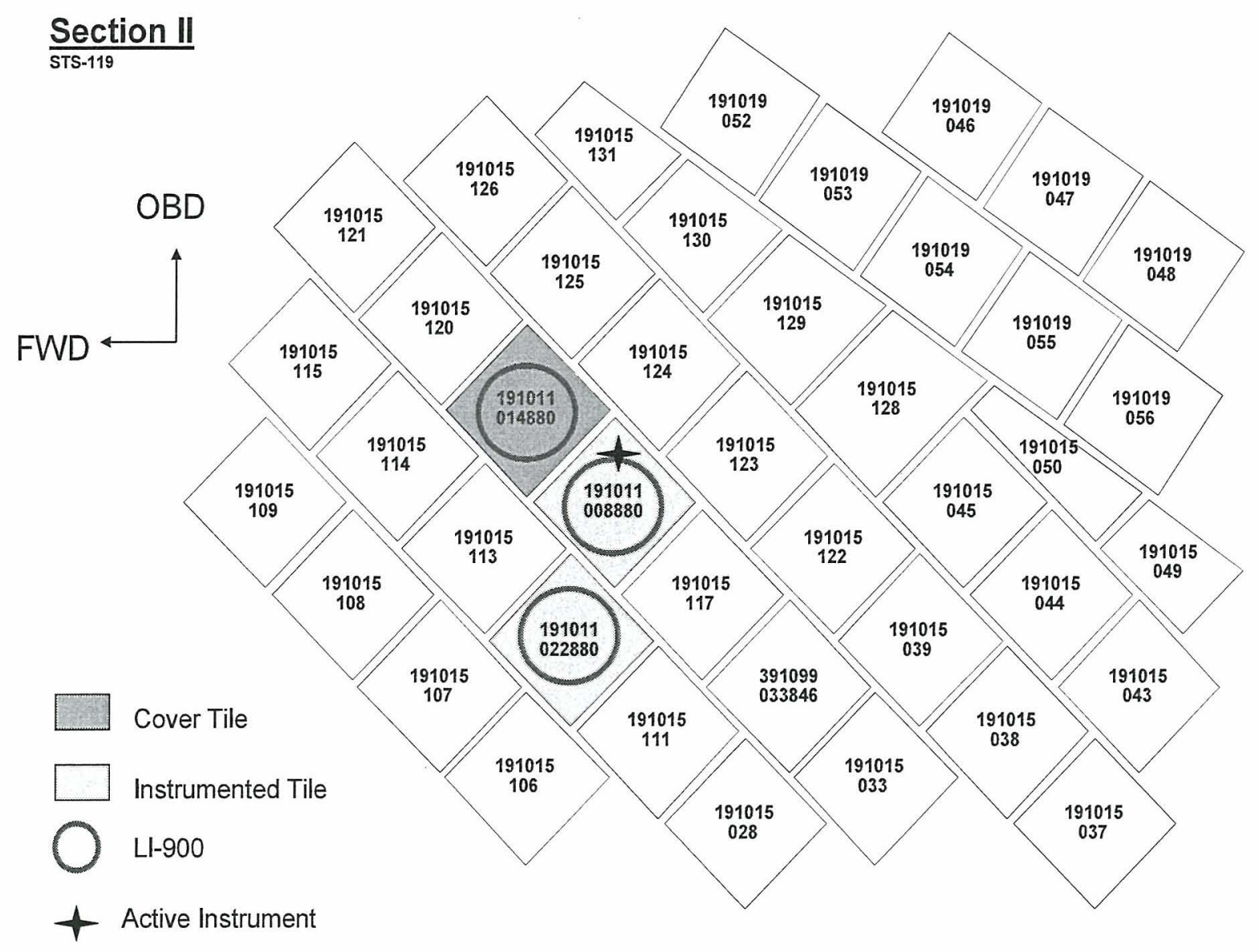

Figure 7. Detailed View of STS-119 Section II Layout 


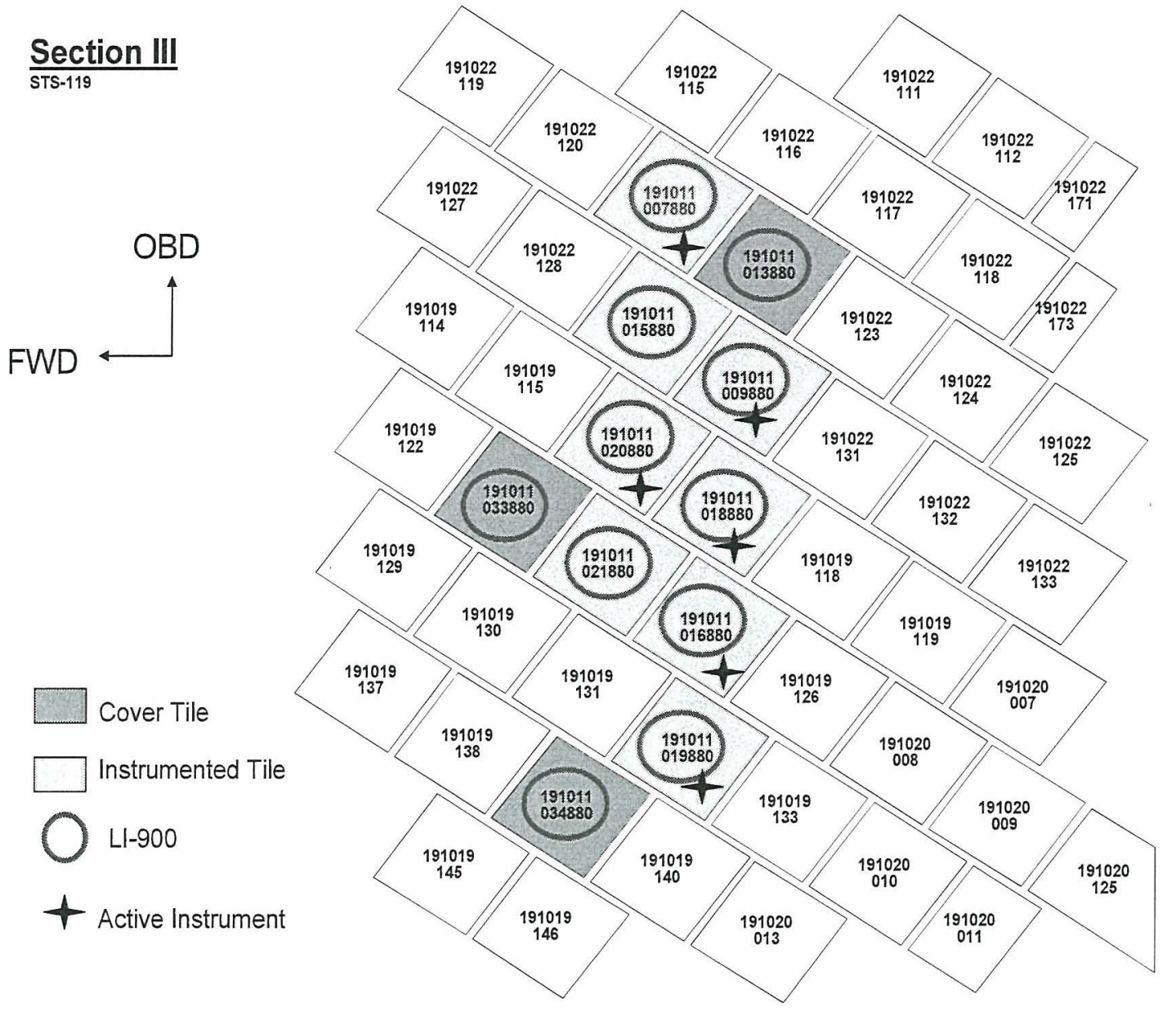

Figure 8. Detailed View of STS-119 Section III Layout 


Table 1. List of Tiles Involved for STS-119 (BLT First Flight)
\begin{tabular}{|c|c|c|c|c|}
\hline Tile Number & Section & T/C Number & Tile Material Type & T/C Active \\
\hline V070-192131-099 & MADS & Control & LI-900 & YES \\
\hline V880-191011-001 & I & Protuberance & BRI-18 & YES \\
\hline V880-191011-036 & I & N/A (Cover Tile) & LI-2200 & N/A \\
\hline V880-191011-040 & I & N/A (Heat) & LI-2200 & N/A \\
\hline V880-191011-041 & I & N/A (Heat) & LI-2200 & N/A \\
\hline V880-191011-035 & I & N/A (Cover Tile) & LI-900 & N/A \\
\hline V880-191011-017 & I & TC \#5 & LI-900 & YES \\
\hline V880-191011-014 & II & N/A (Cover Tile) & LI-900 & N/A \\
\hline V880-191011-008 & II & TC \#2 & LI-900 & YES \\
\hline V880-191011-022 & II & TC \# 9 & LI-900 & NO \\
\hline V880-191011-007 & III & TC \# 1 & LI-900 & YES \\
\hline V880-191011-015 & III & TC \# 3a & LI-900 & NO \\
\hline V880-191011-009 & III & TC \# 3 & LI-900 & YES \\
\hline V880-191011-013 & III & N/A (Cover Tile) & LI-900 & N/A \\
\hline V880-191011-020 & III & TC \# 8 & LI-900 & YES \\
\hline V880-191011-018 & III & TC \# 6 & LI-900 & YES \\
\hline V880-191011-021 & III & TC \# 8a & LI-900 & NO \\
\hline V880-191011-033 & III & N/A (Cover Tile) & LI-900 & N/A \\
\hline V880-191011-016 & III & TC \# 4 & LI-900 & YES \\
\hline V880-191011-019 & III & TC \# 7 & LI-900 & YES \\
\hline V880-191011-034 & III & N/A (Cover Tile) & LI-900 & N/A \\
\hline
\end{tabular}

Discovery landed at Kennedy Space Center on 28 March 2009 completing thirteen days in orbit. The entry data from BLT was reviewed by analysts and the flight experiment was determined to be a success. ${ }^{6}$ Based on the data review, it was decided by the team, and with the concurrence of the Space Shuttle Program that a second flight utilizing a taller $(0.35$ inch) protuberance would provide engineers with data about boundary layer transition behavior approaching Mach 18. From a processing perspective, this required the removal and replacement of several tiles in support of a taller protuberance. ${ }^{6}$

The cover tile (V880-191011-036) and the protuberance tile (V880-191011-001) were removed nondestructively by removing surrounding tiles and allowing technicians to sever the bondline through the SIP. The tiles were sent to the Materials and Processes laboratory in Huntington Beach, California to determine if any significant changes were found in the materials properties. ${ }^{6}$ Streakmarks were noted which were a result of the higher heat loads in the immediate downstream region of the protuberance. This caused the silica molecules to outgas through the RCG in a localized area. This was a typical response and is the reason why the tiles lose their black color over the course of multiple flights. No other notable material differences were found. Based on the streakmark, it was determined to shift TC \#5 slightly inboard in order to correspond to the observed streakline of maximum heating. ${ }^{6}$ 


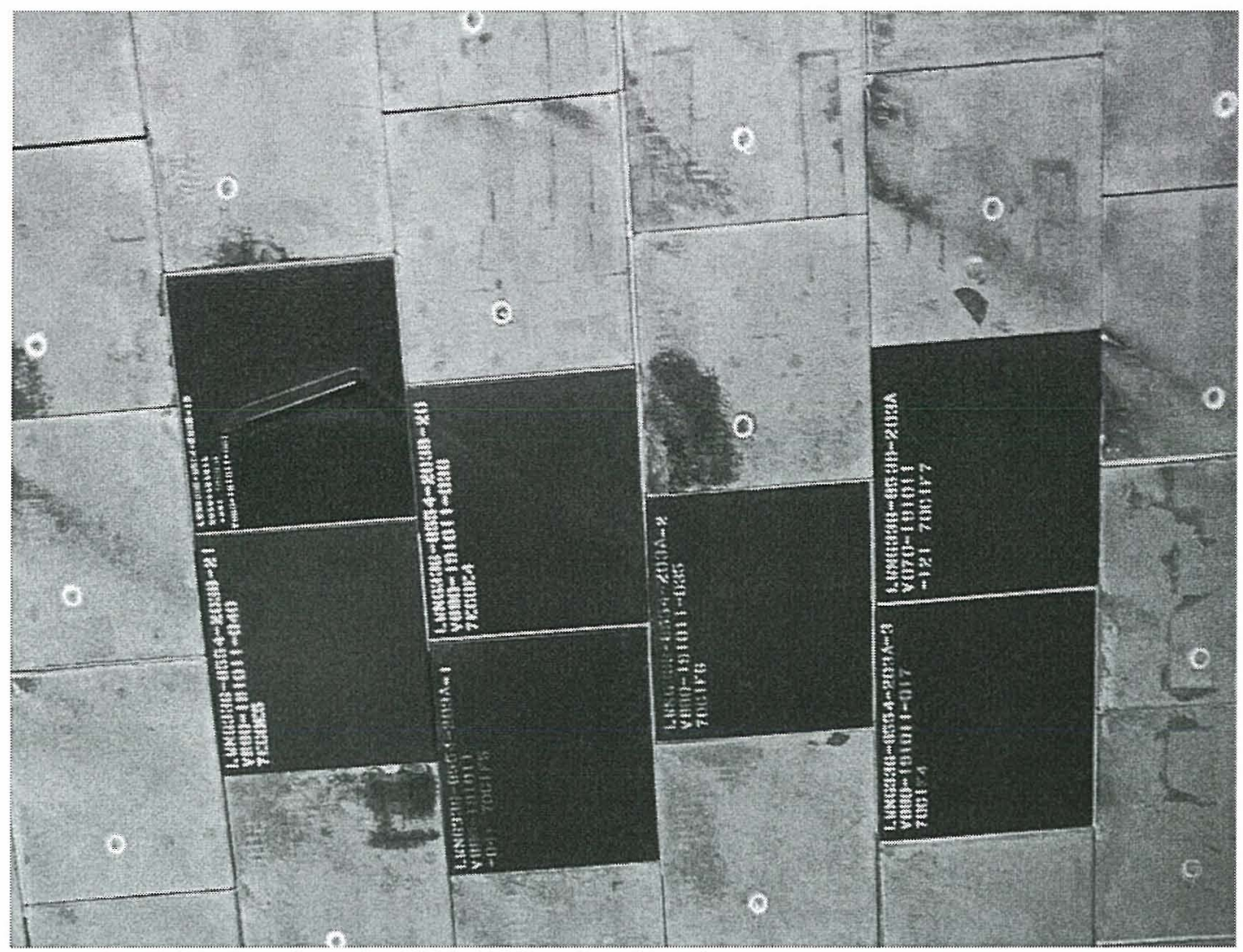

Figure 9. Visible Streakmarks (STS-119 Landing Photo)

Processing for the second flight of the experiment (STS-128) was easier than the first since the majority of the work had already been performed. ${ }^{5}$ What was required on STS-128 processing was the change-out of the protuberance tile itself which was increased to 0.35 inches in height. Additionally, in order to get better resolution of the turbulent wedge stemming from the protuberance and in support of the catalytic coating experiment, analysts decided that TC \#3 and TC \#8 would be deactivated, and TC \#3a and TC \#8a would be activated. This had no impact on tile processing as all work was internal to the vehicle. The extension wire leads were simply removed from the TRJ, capped and stowed, while the thermocouples to be activated were connected and channelized. What had an impact was the change-out of the protuberance itself which drove the cover tile to be removed as well. The cover tile had to be bolstered to an LI-2200 material in order to be able to withstand the higher projected heating produced by a taller protuberance. Sequentially, this required the protuberance to be processed as an instrumented tile while preventing the bonding of the cover tile until the protuberance was checked out. The major issue TPS Engineering encountered during STS-128 processing was trimming the thermocouple wire leads in the protuberance tile. By not trimming the wires early in the process, more opportunity was present that the wires would be warped and possibly kinked during processing. Engineering noted that there was a tight radius on the thermocouple wire, but NDE testing determined that the wire was not kinked as the density of the TC wire was constant. ${ }^{5}$

Unique to the STS-128 mission was the installation of catalytic coating on two of the tiles within the turbulent wedge region. The intent of the catalytic coating experiment was to obtain recombination coefficient data of nitrogen and oxygen on the tile surface in a turbulent hypersonic flow field. ${ }^{6}$ Although it will be the scope of a future paper to discuss the installation and results of the catalytic coating experiment, TPS Engineering had additional requirements to completely fill the tiles surrounding the catalytic coating experiment utilizing ceramic Ames gap fillers. This was not out of the realm of experience, but it was an additional requirement in support of the flight experiments that was met in the shortest processing flow since post-Columbia return to flight.

Figures 10 through 13 and Table 2 show the configuration of STS- 128. 


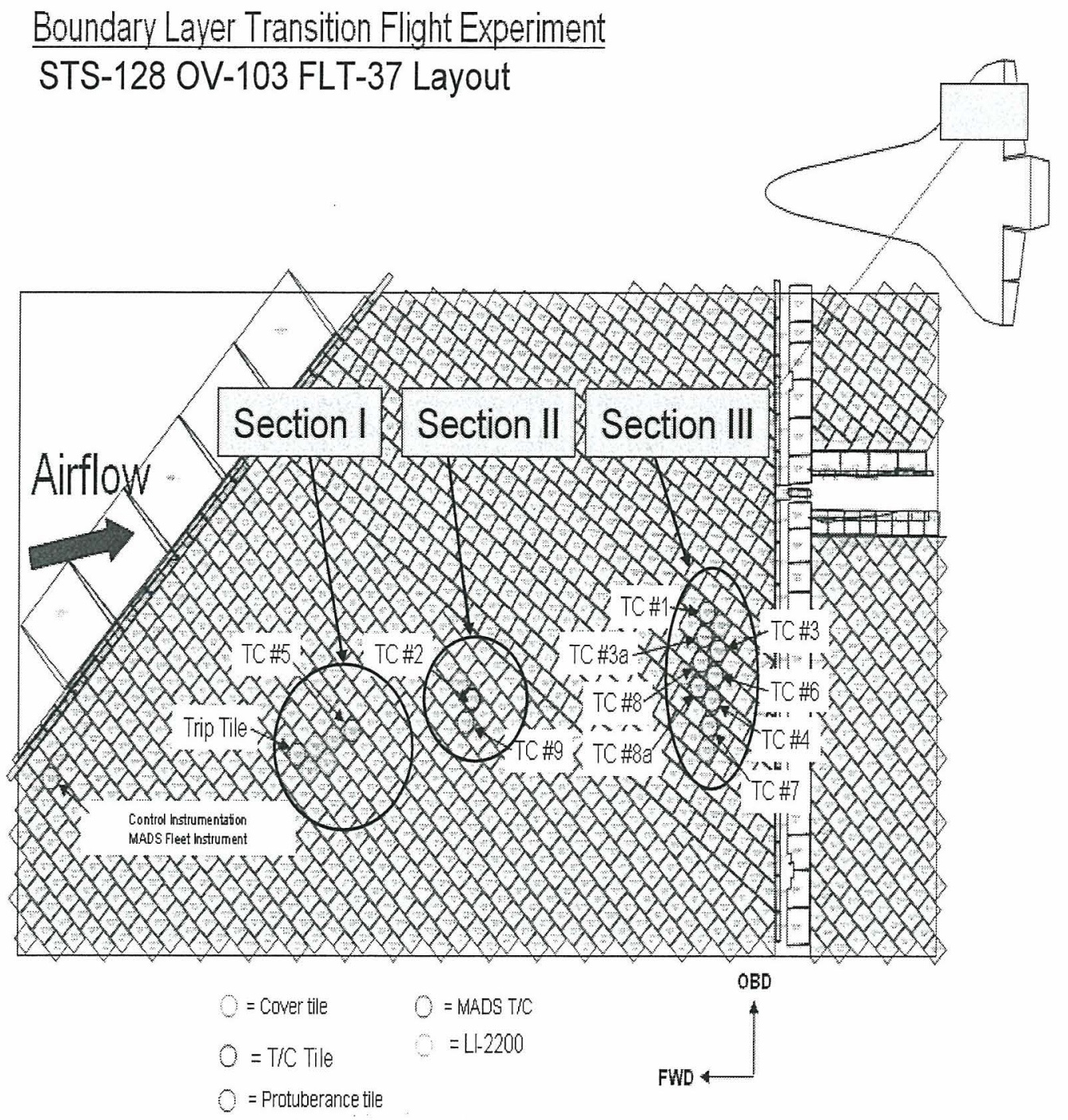

Figure 10. Tile Layout for STS-128 


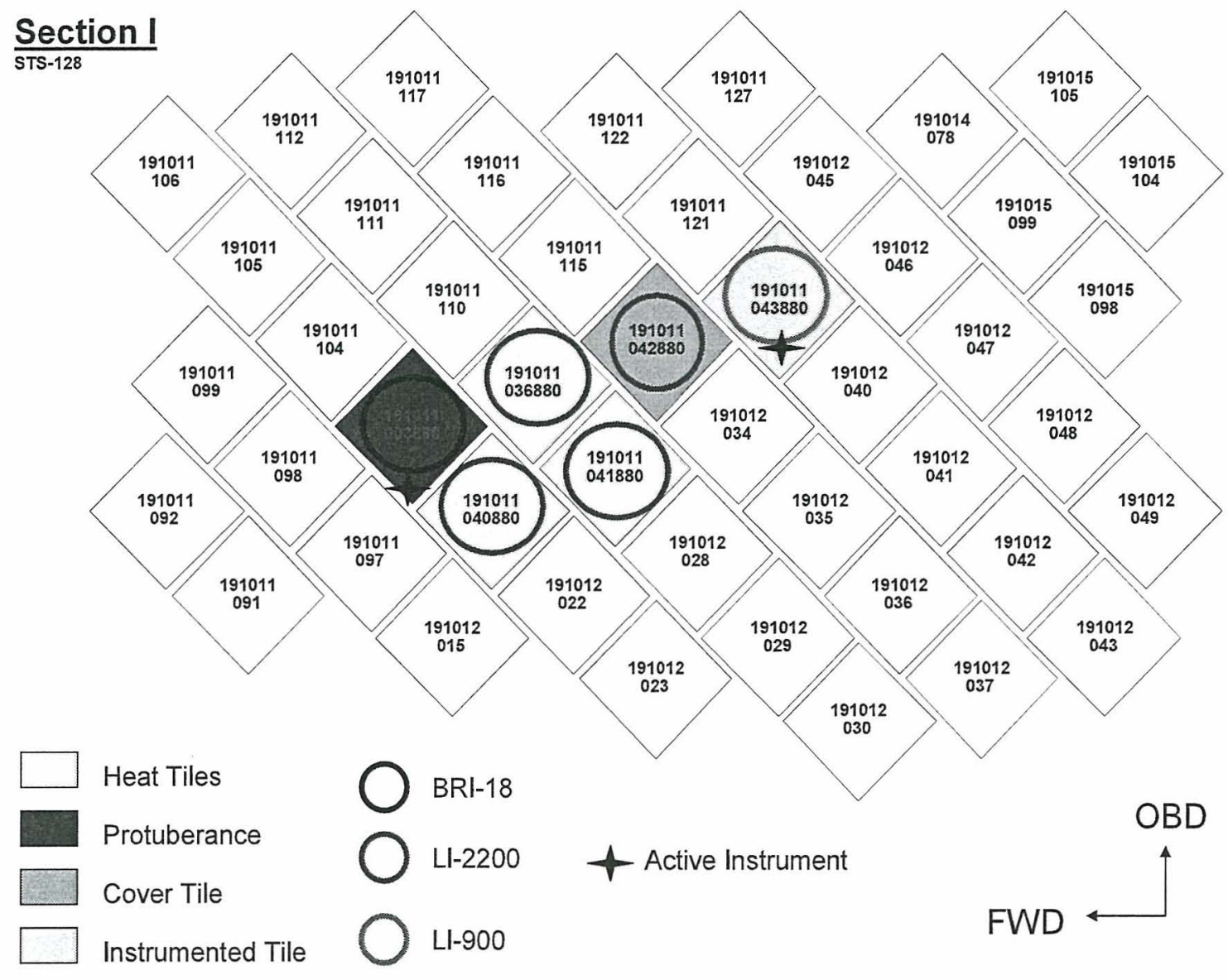

Figure 11. Detailed View of STS-128 Section I Layout 


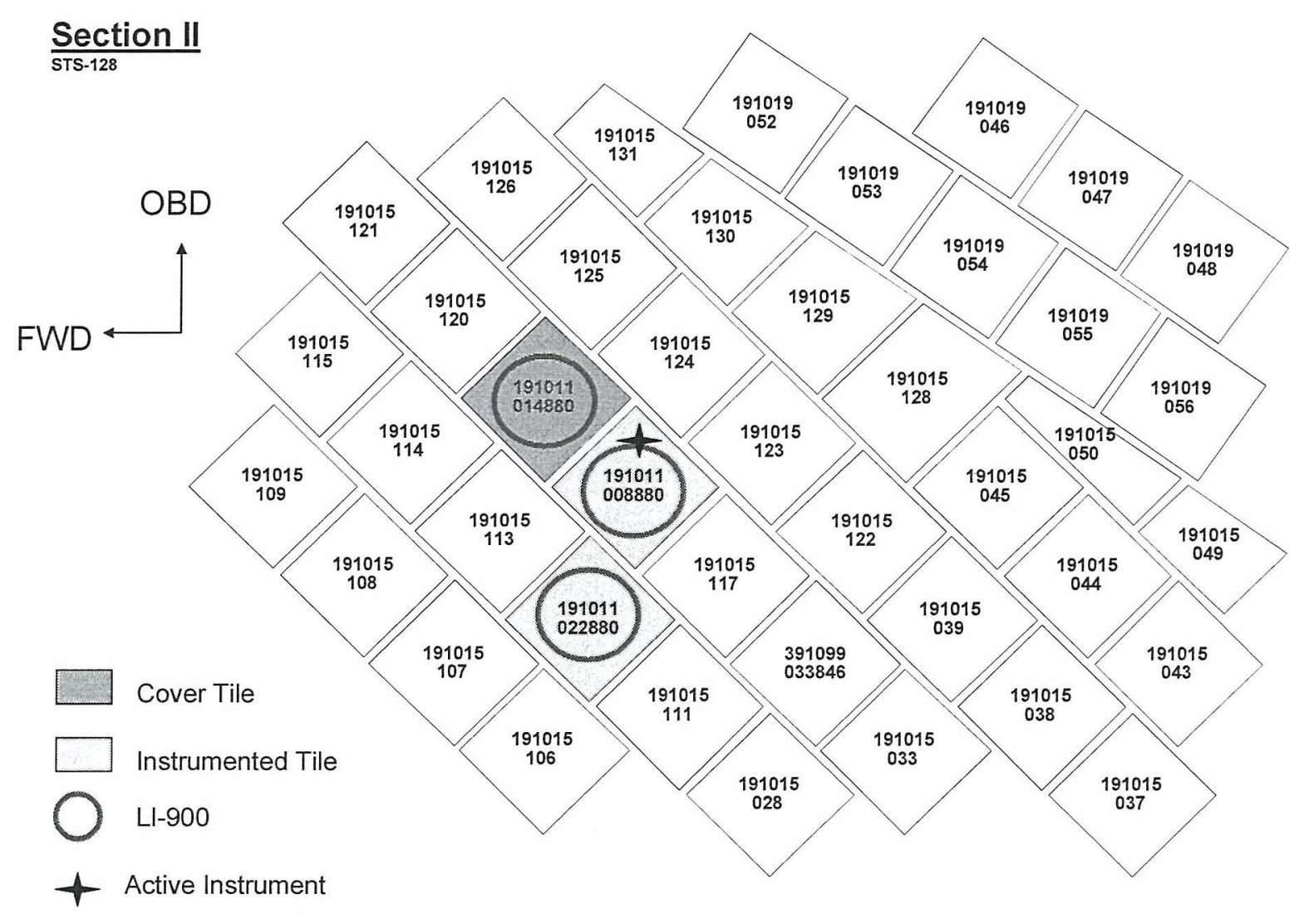

Figure 12. Detailed View of STS-128 Section II Layout 


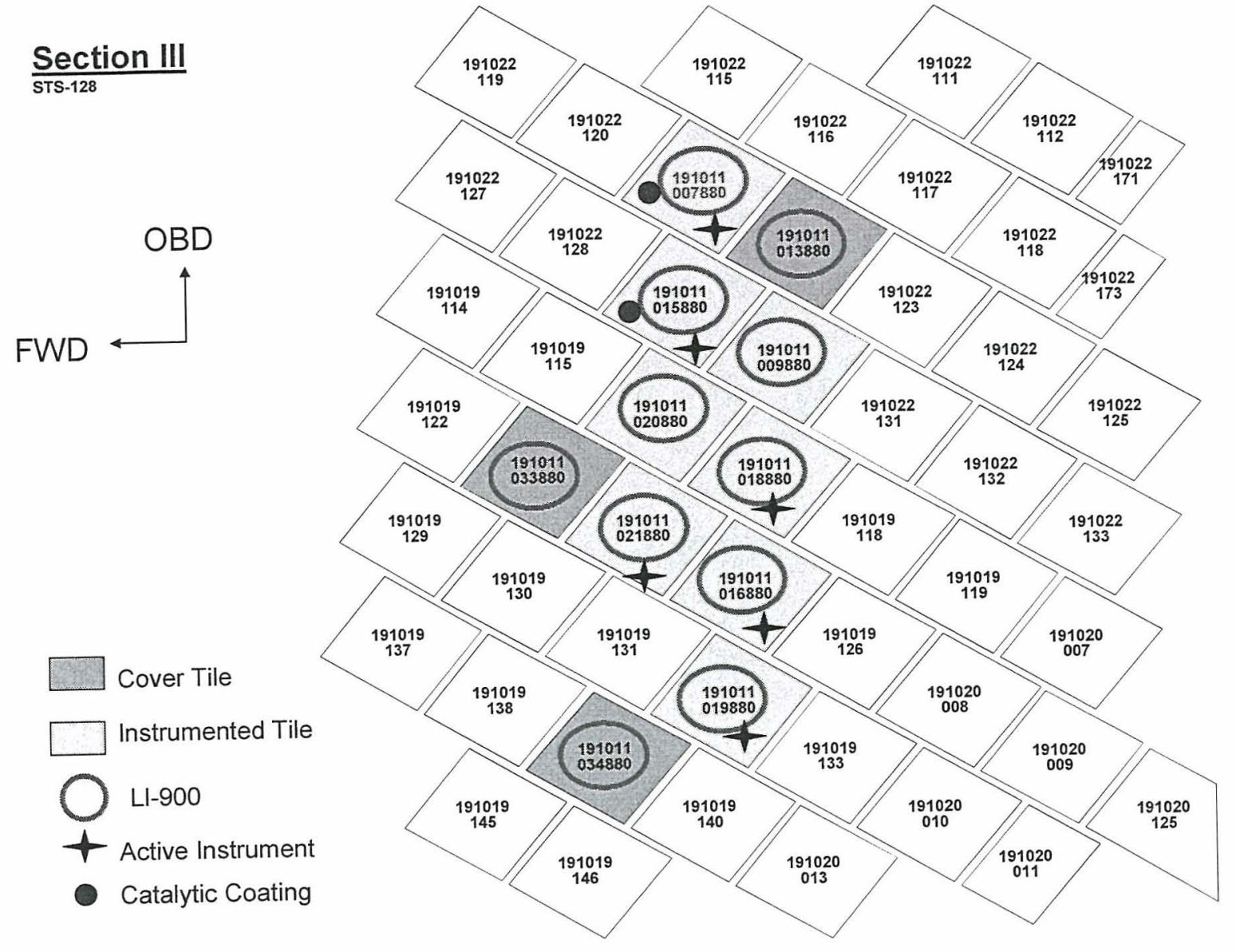

Figure 13. Detailed View of STS-128 Section III Layout 
Table 2. List of Tiles Involved in STS-128 (BLT Second Flight)

\begin{tabular}{|c|c|c|c|c|}
\hline Tile Number & Section & T/C Number & Tile Material Type & T/C Active \\
\hline V070-192131-099 & MADS & Control & LI-900 & YES \\
\hline V880-191011-003 & I & Protuberance & BRI-18 & YES \\
\hline V880-191011-036 & I & N/A (Cover Tile) & LI-2200 & N/A \\
\hline V880-191011-040 & I & N/A (Heat) & LI-2200 & N/A \\
\hline V880-191011-041 & I & N/A (Heat) & LI-2200 & N/A \\
\hline V880-191011-042 & I & N/A (Cover Tile) & LI-2200 & N/A \\
\hline V880-191011-043 & I & TC \#5 & LI-900 & YES \\
\hline V880-191011-014 & II & N/A (Cover Tile) & LI-900 & N/A \\
\hline V880-191011-008 & II & TC \#2 & LI-900 & YES \\
\hline V880-191011-022 & II & TC \# 9 & LI-900 & NO \\
\hline V880-191011-007 & III & TC \# 1 & LI-900 & YES \\
\hline V880-191011-015 & III & TC \# 3a & LI-900 & NO \\
\hline V880-191011-009 & III & TC \# 3 & LI-900 & N/A \\
\hline V880-191011-013 & III & N/A (Cover Tile) & LI-900 & NO \\
\hline V880-191011-020 & III & TC \# 8 & LI-900 & YES \\
\hline V880-191011-018 & III & TC \# 6 & LI-900 & YES \\
\hline V880-191011-021 & III & TC \# 8a & LI-900 & N/A \\
\hline V880-191011-033 & III & N/A (Cover Tile) & LI-900 & YES \\
\hline V880-191011-016 & III & TC \# 4 & LI-900 & YES \\
\hline V880-191011-019 & III & TC \# 7 & N/A \\
\hline V880-191011-034 & III & N/A (Cover Tile) & & \\
\hline
\end{tabular}

Discovery completed STS-128 on 11 September 2009, touching down at Edwards Air Force Base, California after a thirteen day mission to the International Space Station. The BLT data was downloaded from the MADS at Edwards AFB and sent to analysts for review. Although valuable data was obtained during this flight, it was not as clear cut as the data obtained from STS-119. ${ }^{6}$ As a result, the STS-131 mission scheduled for a March 2010 launch will fly a 0.35 inch protuberance tile to obtain a second data point for comparison with STS-128. At the time of the authoring of this paper, the STS-128 protuberance as well as the access tile (V880-191011-036) have both been removed and both are in the process of being manufactured. 3-D measurements of the protuberance itself show that there has been a very small change in geometry. The protuberance had become glossy on the leading edge and at the worst case, has been flattened by 0.016 inches. ${ }^{5}$

Glossiness and the onset of warping shows that transition from laminar to turbulent heat is occurring at speeds approaching Mach 18 which is one objective of the flight experiment. ${ }^{6}$ However, this data also comes with the caveat that aerodynamically, the vehicle forward of the protuberance transitioned asymmetrically and at a higher Mach number than expected (in the case of STS-128, asymmetrical transition means the port side became turbulent significantly earlier than the starboard side). What effect this had on the experiment is currently under review with analysts at Johnson Space Center. It is expected that the third flight (STS-131) will provide the necessary data to validate or refute STS-128 data using a 0.35 inch protuberance.

The Space Shuttle Program may decide to authorize a fourth flight of BLT FE utilizing a 0.5 inch protuberance which may reveal more phenomena of hypersonic flight yet unbeknown to the scientific community. The BLT team hopes that this experimental use of the Space Shuttle Orbiter will benefit future generations of scientists and engineers by providing data in a flight regime that earlier generations coined as "Godspeed." 


\section{References}

${ }^{3}$ Orbiter Entry Boundary Layer Flight Testing, Campbell, Charles et.al. AIAA paper 2006

${ }^{4}$ Callister, William D., Jr. Materials Science and Engineering an Introduction. Fifth Ed., 2000

${ }^{5}$ Spanos, Theodoros Boundary Layer Transition Flight Experiment Implementation on OV-103. Kennedy Engineering Academy Presentation, 2009

${ }^{6}$ Anderson, Brian P. et al, BLT Flight Experiment Overview and In-Situ Measurements, $48^{\text {th }}$ AIAA Aeroscience Meeting Conference 2010

${ }^{7}$ Space Shuttle Transposition Manual, NSTS-1988, August 2000.

${ }^{8}$ McGinley, Catherine B. et al, Review of Orbiter Flight Boundary Layer Transition Data, AIAA.

${ }^{9}$ Discovery Handed Special Heatshield Coating DTO on Behalf of Orion [online] Available from: http://nasa-image.blogspot.com/2009/06/discovery-handed-special-heatshield.html 